\title{
Colour Transversal Vertex Covering Set
}

\section{D.K.Thakkar}

Department of Mathematics, Saurashtra University

Campus, University Road, Rajkot, India

dkthakkarl@yahoo.co.in
V.R.Dave

Shree M. \& N. Virani Science College,

Kalavad Road, Rajkot, India

varadadave@gmail.com

\begin{abstract}
In this paper we introduce new concepts called CTVC set and CTVC number of a graph. We proved that the vertex covering number of a graph is either equal to CTVC number or it is one less than the CTVC number. We have proved some results regarding the effect of removing a vertex from the graph and its effect on the CTVC number of a graph.
\end{abstract}

Keywords: Transversal, Colour Transversal, Vertex Covering Set, Colour Transversal Vertex Covering Set, Dominator Colouring

AMS Subject Classification (2010): 05C15, 05C69.

\section{INTRODUCTION}

The concept of a vertex covering set is well known and has been studied by several authors. The identity $\alpha_{0}(G)+\beta_{0}(G)=|V(G)|\left(\alpha_{0}(G)=\right.$ The vertex covering number $\& \beta_{0}(G)=$ The independence number ) is well known. The concept of colour transversal dominating set was studied in detail in Ph.D. Thesis of Manoharan [7].

We introduce new concepts called CTVC set and CTVC number of a graph. We denote it by $\alpha_{*}(\mathrm{G})$. We prove that $\beta_{0}(\mathrm{G})+\alpha_{*}(\mathrm{G})=\mathrm{n}$ or $\mathrm{n}+1$. Where $\mathrm{n}=$ number of vertices of $\mathrm{G}$. We prove some theorems about removing a vertex from the graph.

We assume that our graphs are finite, simple and undirected. If $G$ is a graph then $V(G)$ will denote the vertex set of $G$ and $E(G)$ will denote the edge set of $G$.

\section{RESUlTS AND DISCUSSION}

\section{Definition2.1 (Colour Transversal Vertex Covering Set)}

Let $\mathrm{G}$ be a graph. A subset $\mathrm{T}$ of $\mathrm{V}(\mathrm{G})$ is said to be a colour transversal vertex covering set of Gif

1. T is a transversal of the colour classes of some chromatic colouring of $\mathrm{G}$ and

2. $\mathrm{T}$ is a vertex covering set of $\mathrm{G}$

This set is also called CTVC set of G.

A CTVC set with minimum cardinality is called a minimum CTVC set or $\alpha_{*}$ set. The cardinality of an $\alpha_{*}$ set is called the colour transversal vertex covering number (or CTVC number) of the graph G and it is denoted as $\alpha_{*}(\mathrm{G})$.

Note that for any graph $G$ and for any chromatic colouring of $G, V(G)$ is always a CTVC set. Thus a CTVC set always exists.

\section{Example 2.2}

Consider the cycle graph $\mathrm{C}_{5}$ with vertices $\mathrm{v}_{1}, \mathrm{v}_{2}, \mathrm{v}_{3}, \mathrm{v}_{4}, \mathrm{v}_{5}$ 


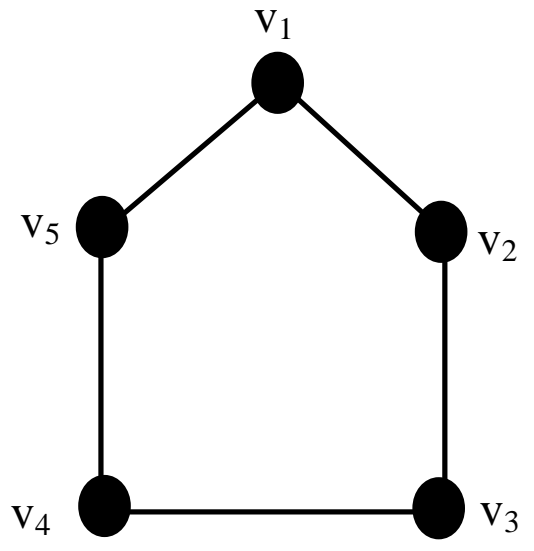

Fig.1

Consider the chromatic colouring which assigns colour -1 to $\mathrm{v}_{1} \& \mathrm{v}_{3}$, colour -2 to $\mathrm{v}_{2} \& \mathrm{v}_{5}$ and colour 3 to $\mathrm{v}_{4}$. Then the set $\mathrm{S}=\left\{\mathrm{v}_{1}, \mathrm{v}_{2}, \mathrm{v}_{4}\right\}$ is a CTVC set of $\mathrm{G}$.

$\therefore \alpha_{*}\left(\mathrm{C}_{5}\right)=3$

Note that $\alpha_{0}\left(\mathrm{C}_{5}\right)=3$

\section{Remark2.3}

We may note that for a given chromatic colouring of $\mathrm{G}$ there may not be a transversal corresponding to colour classes which is an independent set.

In fact it may happen that for any chromatic colouring of $\mathrm{G}$ such a set does not exists.

For example, consider the cycle graph $\mathrm{C}_{5}$ again. In this graph it is impossible to have a set which is a transversal for some chromatic colouring and which is also an independent set. Because in this case a transversal must have atleast three vertices but the size of the maximum independent set of $C_{5}=2$

In general, If for any graph $\mathrm{G}, \quad \beta_{0}(\mathrm{G})<\chi(\mathrm{G})$ then there is no transversal which is an independent set. However it may happen that $\chi(\mathrm{G}) \leq \beta_{0}(\mathrm{G})$ but there is no transversal which is an independent set.

For example, consider the star graph with four vertices

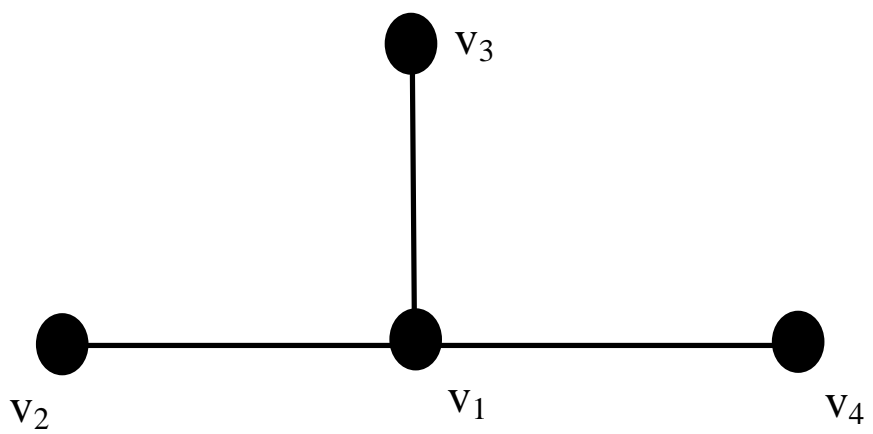

Fig.2

Here, $\chi(\mathrm{G})=2, \quad \beta_{0}(\mathrm{G})=3$

Howeverthere is no transversal which is an independent set.

\section{Example2.4}

Consider the path graphwith four vertices $\mathrm{v}_{1}, \mathrm{v}_{2}, \mathrm{v}_{3}, \mathrm{v}_{4}$

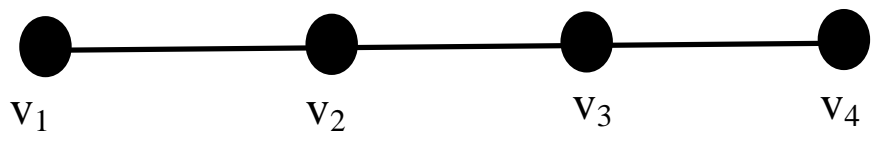

Fig.3 
Consider the chromatic colouring which assigns colour -1 to $\mathrm{v}_{1} \& \mathrm{v}_{3}$ and colour -2 to $\mathrm{v}_{2} \& \mathrm{v}_{4}$. Then obviously the set $\left\{\mathrm{v}_{1}, \mathrm{v}_{4}\right\}$ is a transversal which is also an independent set.

\section{Definition2.5(Dominator Colouring) [3]}

Let $\mathrm{G}$ be a graph. A proper colouring $\mathrm{f}$ of $\mathrm{G}$ is said to be a dominator colouring if every colour class is a single vertex or it is completely dominated by some other colour class.

\section{Definition 2.6(Colour Transversal) [7]}

Let $\mathrm{G}$ be a graph and $\mathrm{C}_{1}, \mathrm{C}_{2}, \ldots \ldots \ldots, \mathrm{C}_{\mathrm{k}}$ be the colour classes of some proper colouring of $\mathrm{G}$. A subset $\mathrm{T}$ of $\mathrm{V}(\mathrm{G})$ is said to be a colour transversal with respect to this colouring if $\mathrm{T} \cap \mathrm{C}_{\mathrm{i}} \neq \phi, \forall \mathrm{i}=1,2$, $\ldots ., \mathrm{k}$

\section{Proposition 2.7}

Let $\mathrm{G}$ be a graph. If a proper colouring of $\mathrm{G}$ is a dominator colouring then there does not exists a colour transversal which is an independent set.

\section{Proof}

Let $\mathrm{G}$ be a graph. Let $\left\{\mathrm{C}_{1}, \mathrm{C}_{2}, \mathrm{C}_{3}, \ldots \ldots \ldots, \mathrm{C}_{\mathrm{k}}\right\}$ be the set of all colour classes corresponding to this proper colouring.

Suppose there is an independent set $S=\left\{\mathrm{v}_{1}, \mathrm{v}_{2}, \ldots \ldots ., \mathrm{v}_{\mathrm{k}}\right\}$ which is a colour transversal of this colour classes. If $\forall \mathrm{i},\left\{\mathrm{v}_{\mathrm{i}}\right\}$ is a colour class then each $\mathrm{v}_{\mathrm{i}}$ is adjacent to each $\mathrm{v}_{\mathrm{j}} \&$ therefore the subgraph induced by the vertices of $\mathrm{S}$ is a complete subgraph.

This is a contradiction.

Therefore there is a colour class say $\mathrm{C}_{1}$ which is not a singleton set. Let $\mathrm{v}$ and $\mathrm{u}$ be two distinct vertices of $\mathrm{C}_{1}$. Then $\mathrm{u}$ is completely dominated by some colour class say $\mathrm{C}_{\mathrm{j}}$. Therefore $\mathrm{u}$ is adjacent to every vertex of $\mathrm{C}_{\mathrm{j}}$. Similarly for every other vertex of $\mathrm{C}_{1}$ this happens.

$\therefore$ It is impossible to get a transversal which is an independent set.

\section{Proposition 2.8 [7]}

Let $\mathrm{G}$ be a graph and $\mathrm{S}$ be an independent subset of $\mathrm{G}$ which is not a maximal independent subset of G. Then there is a chromatic colouring of $G$ in which $V(G)-S$ is a colour transversal for that colouring.

\section{Proof}

Let $\mathrm{f}$ be any chromatic colouring of $\mathrm{G}$. If $\mathrm{V}(\mathrm{G})-\mathrm{S}$ is a colour transversal for this colouring then the result is proved.

So, suppose $V(G)-S$ is not a colour transversal for this colouring. Then there is a colour class $C$ of this colouring such that $\mathrm{C} \subseteq \mathrm{S}$. Now, $\mathrm{S}$ is not a maximal independent set. Therefore $\exists$ a vertex $\mathrm{z}$ which is not in $\mathrm{S}$ and it is not adjacent to any vertex of $\mathrm{S}$. Let $\mathrm{C}^{\prime}$ be the colour class such that $\mathrm{z} \in \mathrm{C}^{\prime}$. Suppose $C^{\prime}=\{z\}$ then $z$ has neighbours in every other colour class. In particular, $z$ has neighbour in C. This implies that $\mathrm{z}$ is adjacent to some vertex of $\mathrm{S}$.

This is a contradiction.

$\therefore \mathrm{C}^{\prime}$ contains atleast two vertices one of which is z. Now define a new colouring $\mathrm{f}$ 'as follows.

$\mathrm{f}^{\prime}(\mathrm{x})=\mathrm{f}(\mathrm{x})$ if $\mathrm{x} \neq \mathrm{z} \quad \& \mathrm{f}^{\prime}(\mathrm{z})=\mathrm{f}(\mathrm{t})$ where $\mathrm{t} \in \mathrm{C}$

Then $\mathrm{f}^{\prime}$ is a chromatic colouring of $\mathrm{f}$ in which $\mathrm{V}(\mathrm{G})-\mathrm{S}$ is a colour transversal.

\section{Theorem 2.9}

Let $G$ be a graph with $n$ vertices. Then either $\beta_{0}(G)+\alpha_{*}(G)=n$ or $\beta_{0}(G)+\alpha_{*}(G)=n+1$

\section{Proof}

Suppose there is a maximum independent set $T$ such that $S=V(G)-T$ is a colour transversal for some chromatic colouring of $\mathrm{G}$. Then $\mathrm{S}$ is a colour transversal vertex covering set of $\mathrm{G}$. 


\section{Claim}

$\mathrm{S}$ is a minimum CTVC set of $\mathrm{G}$

\section{Proof of the Claim}

Suppose $\mathrm{S}$ is not a minimum CTVC set of G. Let $\mathrm{S}_{1}$ be a minimum CTVC set of G.

Then $\left|S_{1}\right|<|S|$

Then $|\mathrm{T}|<\left|\mathrm{V}(\mathrm{G})-\mathrm{S}_{1}\right|$ and $\mathrm{V}(\mathrm{G})-\mathrm{S}_{1}$ is an independent subset of $\mathrm{G}$ because $\mathrm{S}_{1}$ is a vertex covering set of $\mathrm{G}$.

This is a contradiction because $\mathrm{T}$ is a maximum independent subset of $\mathrm{G}$. Thus, $\mathrm{S}$ must be a minimum CTVC set of $G \&$ therefore $\alpha_{*}(G)=|S|$

Obviously, $\beta_{0}(\mathrm{G})+\alpha_{*}(\mathrm{G})=\mathrm{n}$

Suppose for any maximum independent set $\mathrm{T}, \mathrm{V}(\mathrm{G})-\mathrm{T}$ is not a colour transversal for any chromatic colouring of $\mathrm{G}$.

Let $\mathrm{T}$ be any maximum independent subset of $\mathrm{G}$. Let $\mathrm{x} \in \mathrm{T} \&$ consider the set $\mathrm{T}_{1}=\mathrm{T}-\{\mathrm{x}\}$. Then $\mathrm{T}_{1}$ is an independent set which is not maximal.

By the above proposition, there is a chromatic colouring $f$ of $G$ such that $S=V(G)-T_{1}$ is a colour transversal for this colouring. Since $T_{1}$ is an independent set, $\mathrm{S}$ is a vertex covering set. So, $\mathrm{S}$ is a CTVC set.

\section{Claim}

$\mathrm{S}$ is a minimum CTVC set

\section{Proof of the Claim}

Suppose $\mathrm{S}$ is not a minimum CTVC set. Let $\mathrm{S}_{1}$ be a minimum CTVC set of G.

Then $\left|S_{1}\right|<|S|$

Now, let $T^{\prime}=V(G)-S_{1}$. Then $T^{\prime}$ is an independent set $\&\left|T^{\prime}\right|>\left|T_{1}\right|$

Since, $T^{\prime}$ is an independent set $\left|T^{\prime}\right|=\left|T_{1}\right|+1$

$\therefore \mathrm{T}^{\prime}$ is a maximum independent set such that $\mathrm{S}_{1}=\mathrm{V}(\mathrm{G})-\mathrm{T}^{\prime}$ is a colour transversal.

This is a contradiction.

Thus $\mathrm{S}=\mathrm{V}(\mathrm{G})-\mathrm{T}_{1}$ is a minimum CTVC set of $\mathrm{G}$.

i.e. $\alpha_{*}(\mathrm{G})=|\mathrm{S}|$

Note that, $|\mathrm{S}|=\mathrm{n}-\beta_{0}(\mathrm{G})+1$

Thus, $\alpha_{*}(G)=\mathrm{n}-\beta_{0}(\mathrm{G})+1$

$\therefore \alpha_{*}(\mathrm{G})+\beta_{0}(\mathrm{G})=\mathrm{n}+1$

\section{Corollary 2.10}

Let $G$ be a graph. Then, $\alpha_{0}(G)=\alpha_{*}(G)$ or $\alpha_{0}(G)=\alpha_{*}(G)-1$

\section{Proof}

Suppose $\alpha_{*}(G)+\beta_{0}(G)=n$

Also, $\alpha_{0}(\mathrm{G})+\beta_{0}(\mathrm{G})=\mathrm{n}$

$\therefore \alpha_{0}(\mathrm{G})=\alpha_{*}(\mathrm{G})$

Suppose $\alpha_{*}(G)+\beta_{0}(G)=n+1$

$\therefore \alpha_{*}(\mathrm{G})-1+\beta_{0}(\mathrm{G})=\mathrm{n}$

Since, $\alpha_{0}(G)+\beta_{0}(G)=n$

$\alpha_{0}(G)=\alpha_{*}(G)-1$ 


\section{Corollary 2.11}

Let $G$ be a graph. Then $\alpha_{0}(G)=\alpha_{*}(G)$ iff there is a maximum independent set $T$ of $G \ni V(G)-T$ is a colour transversal for some chromatic colouring of $\mathrm{G}$.

\section{Example 2.12}

Consider the cycle graphC $C_{4}$ then $\alpha_{0}(\mathrm{G})=2 \& \alpha_{*}(\mathrm{G})=3$

For this graph, $\alpha_{0}(G)=\alpha_{*}(G)-1$

Consider the cycle graphC $C_{5}$ then $\alpha_{0}(\mathrm{G})=3 \& \alpha_{*}(\mathrm{G})=3$

For this graph, $\alpha_{0}(\mathrm{G})=\alpha_{*}(\mathrm{G})$

\section{Note 2.13 (Vertex Removal from a Graph)}

Let $\mathrm{G}$ be a graph and $\mathrm{v} \in \mathrm{V}(\mathrm{G})$. Consider the subgraph $(\mathrm{G}-\mathrm{v})$ also consider the numbers $\alpha_{*}(\mathrm{G})$ $\& \alpha_{*}(\mathrm{G}-\mathrm{v})$

We may ask the following question

What is the relation between $\alpha_{*}(\mathrm{G}) \& \alpha_{*}(\mathrm{G}-\mathrm{v})$ ?

We have the following proposition.

\section{Theorem 2.14}

Let $\mathrm{G}$ be a graph and $\mathrm{v} \in \mathrm{V}(\mathrm{G})$. Suppose $\chi(\mathrm{G}-\mathrm{v})=\chi(\mathrm{G})$ then $\alpha_{*}(\mathrm{G}-\mathrm{v}) \leq \alpha_{*}(\mathrm{G})$

\section{Proof}

Let $\mathrm{S}$ be a minimum CTVC set of $\mathrm{G}$ with respect to some chromatic coloring $\mathrm{f}$ of $\mathrm{G}$. Since $\chi(\mathrm{G}-\mathrm{v})=$ $\chi(\mathrm{G}),\{\mathrm{v}\}$ is not a colour class for this chromatic colouring of $\mathrm{G}$. Consider the function $\mathrm{g}$ which is restriction of $\mathrm{f}$ on $\mathrm{G}-\mathrm{v}$ then $\mathrm{g}$ is a chromatic colouring of $\mathrm{G}-\mathrm{v}$ because $\chi(\mathrm{G}-\mathrm{v})=\chi(\mathrm{G})$.

Case 1:Suppose $v \notin S$

Then obviously $\mathrm{S}$ is a colour transversal for the chromatic colouring $\mathrm{g}$ of $(\mathrm{G}-\mathrm{v})$ because $\mathrm{g}$ uses the same colours as the $\mathrm{f}$.

Also $S$ is a vertex covering set of $(G-v)$.

$\therefore \mathrm{S}$ is a CTVC set of $(\mathrm{G}-\mathrm{v})$ (w.r.t. the chromatic colouring g)

$\therefore \alpha_{*}(\mathrm{G}-\mathrm{v}) \leq|\mathrm{S}|=\alpha_{*}(\mathrm{G})$

Case 2: Suppose $v \in S$

Then $S-\{v\}$ is a vertex covering set of $(G-v)$ but it need not be a colour transversal w.r.t. the colouring $g$. Let $u$ be a vertex of $G-v$ which has the same colour as $v(\{v\}$ is not a colour class in $f)$.

Let $S_{1}=(S-\{v\}) \cup\{u\}$

Then $S_{1}$ is a CTVC set of $(G-v)$.

$\therefore \alpha_{*}(\mathrm{G}-\mathrm{v}) \leq\left|\mathrm{S}_{1}\right|=|\mathrm{S}|=\alpha_{*}(\mathrm{G})$

\section{Remark 2.15}

It can be observed from example -1 that $\alpha_{*}\left(C_{5}\right)=3$ while $\alpha_{*}\left(C_{5}-v_{1}\right)=2, \alpha_{*}\left(C_{5}-v_{5}\right)=2$

Here, $\alpha_{*}(G-v)<\alpha_{*}(G)$

It can be observed from example -2 that $\alpha_{*}\left(\mathrm{P}_{4}\right)=2$ while $\alpha_{*}\left(\mathrm{P}_{4}-\mathrm{v}_{1}\right)=2, \alpha_{*}\left(\mathrm{P}_{4}-\mathrm{v}_{2}\right)=2$

Here, $\alpha_{*}(\mathrm{G}-\mathrm{v})=\alpha_{*}(\mathrm{G})$

\section{Theorem 2.16}

Let $\mathrm{G}$ be a graph and $\mathrm{v} \in \mathrm{V}(\mathrm{G})$. If $\alpha_{*}(\mathrm{G}-\mathrm{v})<\alpha_{*}(\mathrm{G})$ then $\alpha_{*}(\mathrm{G}-\mathrm{v})=\alpha_{*}(\mathrm{G})-1$

\section{Proof}

Suppose that $\chi(\mathrm{G}-\mathrm{v})<\chi(\mathrm{G})$ then $\chi(\mathrm{G}-\mathrm{v})=\chi(\mathrm{G})-1$ 
Let $S_{1}$ be minimum CTVC set of $(G-v)$ with respect to some chromatic colouring $f$ of $(G-v)$. Suppose this colouring has used colours $1,2, \ldots . ., \mathrm{k}-1$.

If we assign any of this colour to $\mathrm{v}$ then it will not be a proper colouring because $\chi(\mathrm{G}-\mathrm{v})<\chi(\mathrm{G})$.

Therefore a new colour says $k$ must be assigned to vertex $v$ to get a new chromatic colouring $f^{\prime}$ of $G$ as follows

$\mathrm{f}^{\prime}(\mathrm{v})=\mathrm{k}$ and

$\mathrm{f}^{\prime}(\mathrm{w})=\mathrm{f}(\mathrm{w})$ if $\mathrm{w} \neq \mathrm{v}$

The set $S_{1}$ may or may not be a vertex covering set of $G$ but it is certainly not a colour transversal for this colouring $f$ ' of $G$. Also it can not be a colour transversal for any chromatic colouring of $G$ because it will imply that the chromatic number of $\mathrm{G}=\mathrm{k}-1$.

If $S=S_{1} \cup\{v\}$ then $S$ is both a colour transversal \& a vertex covering set of $G$.

Since $\alpha_{*}(G-v)<\alpha_{*}(G)$, S must be a minimum CTVC set of G.

$\therefore \alpha_{*}(\mathrm{G})=|\mathrm{S}|=\left|\mathrm{S}_{1}\right|+1=\alpha_{*}(\mathrm{G}-\mathrm{v})+1$

Now suppose $\chi(\mathrm{G}-\mathrm{v})=\chi(\mathrm{G})$

Let $S_{1}$ be minimum CTVC set of $(G-v)$ with respect to some chromatic colouring $f$ of $(G-v)$.

Since $\chi(G-v)=\chi(G),\{v\}$ is not a colour class in any chromatic colouring of $G$.

Let $g$ be a chromatic colouring of $G \ni$ the restriction of $G$ on $(G-v)$ is the chromatic colouring $f$. In this colouring the colour of $\mathrm{v}$ will also appear as colour of some other vertex of $\mathrm{G}$.

$\therefore \mathrm{S}_{1}$ is a colour transversal for this colouring $\mathrm{g}$.

Since $\alpha_{*}(G-v)<\alpha_{*}(G), S_{1}$ can not be a vertex covering set of $G$. Let $S=S_{1} \cup\{v\}$ then obviously $S$ is a vertex covering set of $\mathrm{G}$ and it is also a colour transversal with respect to chromatic colouring $\mathrm{g}$ of G. Since $\alpha_{*}(G-v)<\alpha_{*}(G)$, the set $S$ must be minimum.

Thus, $\alpha_{*}(\mathrm{G})=|\mathrm{S}|=\left|\mathrm{S}_{1}\right|+1=\alpha_{*}(\mathrm{G}-\mathrm{v})+1$

\section{Proposition 2.17}

Let $G$ be a graph and $v \in V(G)$. If $\alpha_{*}(G-v)<\alpha_{*}(G)$ and $\alpha_{*}(G)=\alpha_{0}(G)$ then $\alpha_{0}(G-v)<\alpha_{0}(G)$

\section{Proof}

Suppose $\alpha_{0}(G-v)=\alpha_{0}(G)$

Now, $\alpha_{*}(G-v)=\alpha_{*}(G)-1$

$$
\begin{gathered}
=\alpha_{0}(\mathrm{G})-1 \\
<\alpha_{0}(\mathrm{G})=\alpha_{0}(\mathrm{G}-\mathrm{v}) \\
\therefore \alpha_{*}(\mathrm{G}-\mathrm{v})<\alpha_{0}(\mathrm{G}-\mathrm{v})
\end{gathered}
$$

This is a contradiction

$\therefore \alpha_{0}(\mathrm{G}-\mathrm{v})<\alpha_{0}(\mathrm{G})$

\section{Corollary 2.18}

Let $\mathrm{G}$ be a graph and $\mathrm{v} \in \mathrm{V}(\mathrm{G})$. If $\alpha_{0}(\mathrm{G}-\mathrm{v})<\alpha_{0}(\mathrm{G}) \& \alpha_{0}(\mathrm{G})<\alpha_{*}(\mathrm{G})$ then $\alpha_{*}(\mathrm{G}-\mathrm{v})<\alpha_{*}(\mathrm{G})$

\section{Proof}

Suppose $\alpha_{*}(\mathrm{G}-\mathrm{v})=\alpha_{*}(\mathrm{G})$

Then, $\alpha_{*}(\mathrm{G}-\mathrm{v})=\alpha_{*}(\mathrm{G})>\alpha_{0}(\mathrm{G})>\alpha_{0}(\mathrm{G}-\mathrm{v})$

Now, $\alpha_{0}(\mathrm{G})=\alpha_{*}(\mathrm{G})-1$ and $\alpha_{0}(\mathrm{G}-\mathrm{v})=\alpha_{0}(\mathrm{G})-1$

$\therefore \alpha_{0}(\mathrm{G}-\mathrm{v})=\alpha_{*}(\mathrm{G}-\mathrm{v})-2$ 
Which is not possible

$\therefore \alpha_{*}(\mathrm{G}-\mathrm{v})<\alpha_{*}(\mathrm{G})$

\section{Proposition 2.19}

If $\alpha_{*}(G-v)>\alpha_{*}(G)$ then $\alpha_{0}(G-v)=\alpha_{0}(G)=\alpha_{*}(G)$

Proof

First we prove that $\alpha_{0}(\mathrm{G})=\alpha_{*}(\mathrm{G})$

Suppose $\alpha_{0}(G)<\alpha_{*}(G)$

Then $\alpha_{*}(G-v)-\alpha_{0}(G-v)=\alpha_{*}(G-v)-\alpha_{*}(G)+\alpha_{*}(G)-\alpha_{0}(G)+\alpha_{0}(G)-\alpha_{0}(G-v)$

$$
\geq 1+1+0=2
$$

$\therefore \alpha_{*}(\mathrm{G}-\mathrm{v})-\alpha_{0}(\mathrm{G}-\mathrm{v}) \geq 2$

Which is not possible. Thus, $\alpha_{0}(G)=\alpha_{*}(G)$

Suppose $\alpha_{0}(G-v)<\alpha_{0}(G)$

Then $\alpha_{*}(G-v)-\alpha_{0}(G-v)=\alpha_{*}(G-v)-\alpha_{*}(G)+\alpha_{*}(G)-\alpha_{0}(G-v)\left(\because \alpha_{0}(G)=\alpha_{*}(G)\right)$

$\geq 1+1=2$

Again this is a contradiction.

$\therefore \alpha_{0}(\mathrm{G}-\mathrm{v})=\alpha_{0}(\mathrm{G})$

\section{Remark 2.20}

From the above proposition it follows that if $\alpha_{*}(G-v)>\alpha_{*}(G)$ then every minimum CTVC set of $G$ does not contain $\mathrm{v}$ because such a set is always a minimum vertex covering set of $\mathrm{G}$ $\left(\because \alpha_{0}(G)=\alpha_{*}(G)\right.$ is a minimum vertex covering set of $G$ and since $\alpha_{0}(G-v)=\alpha_{0}(G)$ no minimum vertex covering set can contain vertex v.)

\section{Concluding Remark}

We have proved in theorem -2 that if $\chi(\mathrm{G}-\mathrm{v})=\chi(\mathrm{G})$ then $\alpha_{*}(\mathrm{G}-\mathrm{v}) \leq \alpha_{*}(\mathrm{G})$ however we do not know if $\chi(\mathrm{G}-\mathrm{v})<\chi(\mathrm{G})$ then $\alpha_{*}(\mathrm{G}-\mathrm{v}) \leq \alpha_{*}(\mathrm{G})$.

We Present the following conjecture.

\subsection{Conjecture}

If $\chi(G-v)<\chi(G)$ then $\alpha_{*}(G-v) \leq \alpha_{*}(G)$.

\section{REFERENCES}

[1] Thakkar D. and Bosamiya J., Graph Critical with respect to Independent Domination, Journal of Discrete Mathematical Sciences \& Cryptography 16,179-186,(2013).

[2] Thakkar D. and Bosamiya J., Vertex Covering Number of a Graph, Mathematics Today 27,30-35 (2011).

[3] GeraR., Horton S. and RasmussenC., Dominator Colorings and Safe Clique Partitions, Congress Num. 181, 19 - 32(2006).

[4] West D. , Introduction to Graph Theory, 2nd Edition, Pearson Education, India, (2001)

[5] HaynesT., HedetniemiS. andSlaterP., Domination in Graphs Advanced Topics, Marcel Dekker, Inc., New York, (1998).

[6] HaynesT., HedetniemiS. andSlaterP., Fundamental of Domination in Graphs, Marcel Dekker, Inc., New York, (1998)

[7] Manoharan R., Dominating Colour Transversals in Graphs, Ph.D. Thesis, Bharathidasan University, India, (2009) 


\section{AUTHORS' BIOGRAPHY}

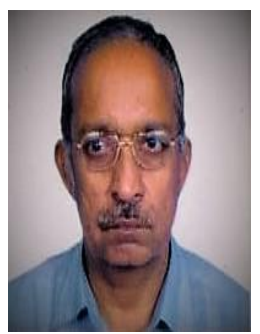

Mr.D.K.Thakkar, is in the Department of Mathematics of Saurashtra University, Rajkot. His areas of interest are Graph Theory, Topology and Discrete Mathematics. He has published over 45 research papers in various journals.

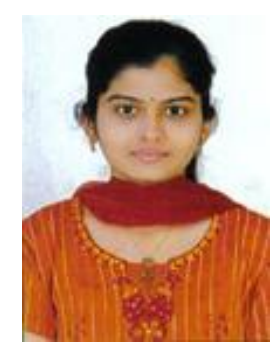

Ms. V. R. Dave, is a young research student who likes to work in a challenging environment. She is working as an Assistant Professor in Shree M. and N. Virani Science College. Her area of interest is Graph Theory. 